\title{
Firearm Use in G- and PG-Rated Movies, 2008-2012
}

\author{
Andrew R. Pelletier, MD, MPH, Jon Eric Tongren, PhD, MSPH, and Julie Gilchrist, MD \\ CDC, Atlanta, Georgia \\ Andrew R. Pelletier: arp1@cdc.gov
}

\section{Introduction}

Popular movies represent a common form of media exposure for children, whether viewed in theaters, on TV, or over the Internet. Based on social cognitive theory, children learn behaviors in part through their exposure to media images. ${ }^{1}$ Exposure to violence in media may have a negative impact on children. ${ }^{2}$ From 1995 to 2007, almost a third (31\%) of the Gand PG-rated movies with the highest U.S. box-office gross revenues had scenes involving firearms. ${ }^{3-5}$ Movies released during 2008-2012 were examined to determine whether the depiction of firearms in movies marketed to children has changed.

\section{Methods}

The original protocol from the 1995-1997 study was used. ${ }^{3,6}$ For each year from 2008 through 2012, the 25 G- or PG-rated movies with the highest annual domestic box-office gross revenues were identified, totaling 125 movies in all. Movies or scenes were excluded if they were animated, not set in the present day, or documentaries. The coding unit was a person-scene, defined as a scene in which one person was shown with a firearm. If two characters in a scene both had firearms, then that would constitute two person-scenes. Possession or handling of firearms was recorded only for characters with speaking roles. All movies were watched in DVD format by the same person. Comparisons between previous studies and 2008-2012 data were analyzed in 2013 by two-sided chi-square tests for trend using EpiInfo, version 3.3, and the Mann-Kendall trend test. Differences were considered significant at $p<0.05$.

\section{Results}

Of 125 movies, 56 (45\%) met the study inclusion criteria, with five (9\%) G-rated movies and $51(91 \%)$ PG-rated movies. Nineteen (34\%) movies depicted characters with firearms (Table 1). Ninety-four person-scenes depicted characters with firearms, with a median of two person-scenes per movie (range, 1-16). Four movies accounted for 59 (63\%) personscenes with firearms.

Address correspondence to: Andrew R. Pelletier, MD, MPH, CDC Botswana/American Embassy, 2170 Gaborone Place, Dulles VA 20189-2170.

No financial disclosures were reported by the authors of this paper. 
Of characters with firearms, all were adults; ninety-two (98\%) were male. Sixty (64\%) characters with firearms were involved in law enforcement or security (e.g., police officers, soldiers); 23 (24\%) were criminals; and 11 (12\%) were other characters (e.g., parents, cowboys). Of person-scenes involving firearms, ten (11\%) involved fantasy characters (e.g., miniature people and visitors from another planet). Fifty-seven (61\%) person-scenes depicted characters handling firearms, and $36(38 \%)$ person-scenes depicted characters making a threatening gesture with a firearm. Twelve (13\%) characters discharged a firearm: seven at a person, four at an inanimate object, and one into the air. Two (2\%) person-scenes depicted characters injured by gunfire including one person who was killed.

In examining trends over time, the number of movies in which a character made a threatening gesture with a firearm declined significantly as did the number of person-scenes involving the handling of firearms. No other changes were statistically significant.

\section{Discussion}

Firearms continue to be shown frequently in G- and PG-rated movies, though there is evidence of declines in certain depictions such as movies in which a character makes a threatening gesture with a firearm and person-scenes in which a firearm is handled. As noted previously, movies rarely showed the consequences of firearm use, including injury and death. ${ }^{3-5}$ In the movies examined from 1995 through 2012, there were a total of 82 personscenes in which a firearm was discharged; only eight (10\%) of these scenes resulted in an injury. These types of portrayals may cause children to minimize the hazards of risky behaviors. $^{7}$

This study had at least three limitations. First, the number of movies and person-scenes in selected firearm categories was small, which restricted our ability to detect statistically significant changes from previous studies. Second, including only scenes for characters with speaking roles underestimated the number of person-scenes that children viewed, as movies often depicted nonspeaking characters with firearms. Third, no test of the reliability of data collection was conducted.

Although a direct relationship between media violence and actual firearm violence has not been established, there is evidence of an association between media violence and some measures of aggression and violent behavior. ${ }^{8}$ Parents should be aware that G- and PG-rated movies still frequently depict firearms. Health providers caring for children should provide counseling on violence prevention and media exposure. ${ }^{9,10}$ The entertainment industry should consider the impact of how firearms are depicted in children's movies.

\section{Acknowledgments}

There was no external funding for this study. The work was done by federal employees of CDC. The study protocol was approved by CDC; the manuscript describing the results of the study was cleared by CDC. The findings and conclusions in this report are those of the authors and do not necessarily represent the official position of CDC. 


\section{References}

1. Bandura A. Health promotion by social cognitive means. Health Educ Behav. 2004; 31(2):143-164. [PubMed: 15090118]

2. Council on Communications and Media. Policy statement—media violence. Pediatrics. 2009; 124(5):1495-1503. [PubMed: 19841118]

3. Pelletier AR, Quinlan KP, Sacks JJ, Van Gilder TJ, Gilchrist J, Ahluwalia HK. Firearm use in Gand PG-rated movies. JAMA. 1999; 281(5):428. [PubMed: 10442653]

4. Ramsey LT, Pelletier AR. Update on firearm use in G- and PG-rated movies. JAMA. 2004; 292(23): 2836-2837. [PubMed: 15598913]

5. Tongren JE, Sites A, Zwicker K, Pelletier A. Firearm use in G- and PG-rated movies, 2003-2007. JAMA. 2009; 301(21):2213-2214. [PubMed: 19491182]

6. Pelletier AR, Quinlan KP, Sacks JJ, Van Gilder TJ, Gilchrist J, Ahluwalia HK. Injury prevention practices as depicted in G-rated and PG-rated movies. Arch Pediatr Adolesct Med. 2000; 154(3): 283-286.

7. Winston FK, Duyck Woolf K, Jordan A, Bhatia E. Actions without consequences: injury-related messages in children's programs. Arch Pediatr Adolesc Med. 2000; 154(4):366-369. [PubMed: 10768674]

8. IOM and National Research Council. Priorities for research to reduce the threat of firearm-related violence. Washington DC: National Academies Press; 2013.

9. Committee on Injury, Violence, and Poison Prevention. Policy statement-Role of the pediatrician in youth violence prevention. Pediatrics. 2009; 124(1):393-402. [PubMed: 19520726]

10. Council on Communications and Media. Policy statement—children, adolescents, and the media. Pediatrics. 2013; 132(5):958-961. 

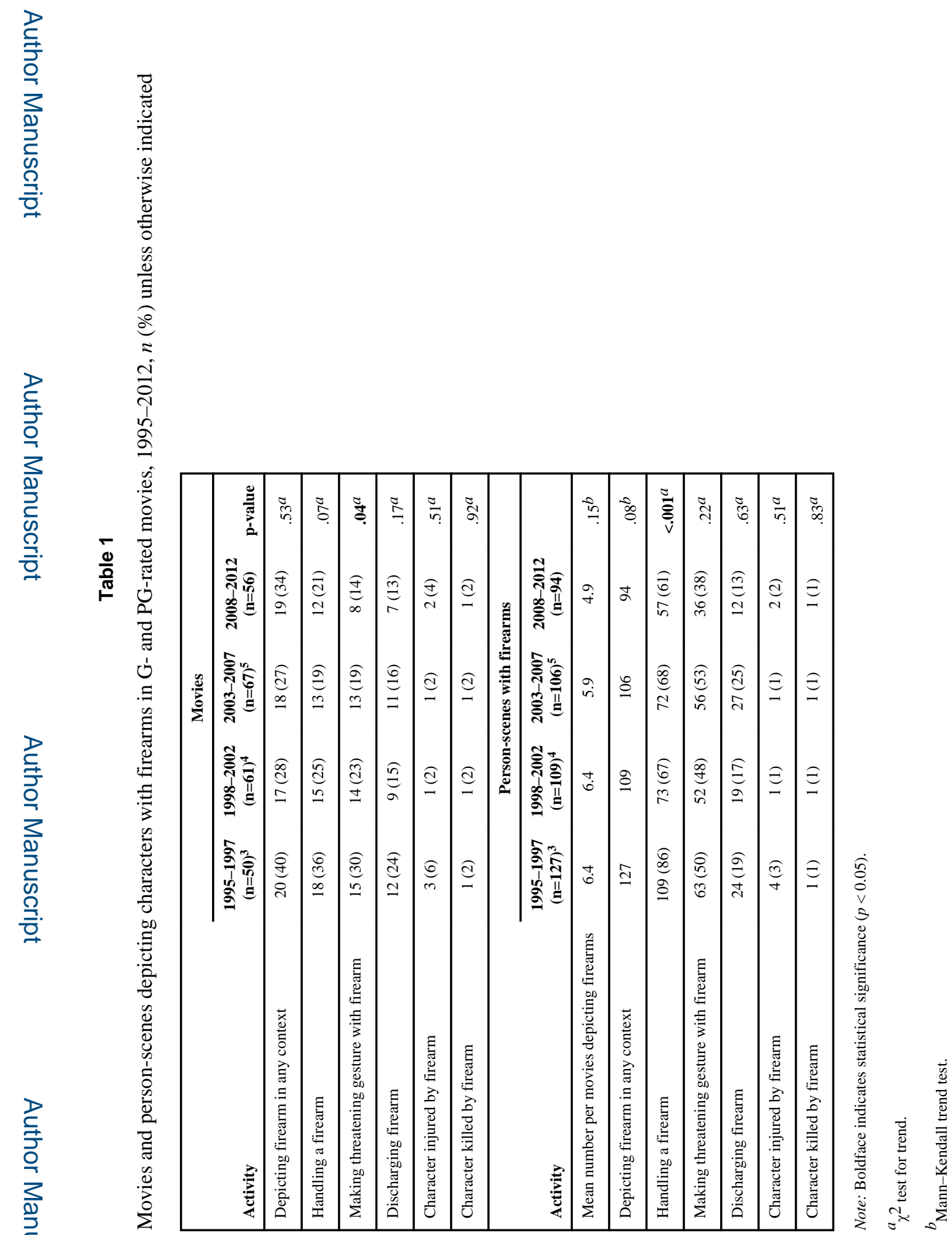

Am J Prev Med. Author manuscript; available in PMC 2016 February 22. 